\section{Stationäre Rehabilitation bei Abhängigkeitserkrankungen - Strukturelle Aspekte}

Klaus Huck-Langer, Brigitte Maier-Riehle, Ferdinand Schliehe

Rehabilitationswissenschaftliche Abteilung, Verband Deutscher Rentenversicherungsträger
Zusammenfassung: Aufbauend auf einem Überblick über die von der Rentenversicherung erhobene Reha-Statistik für den Bereich der Entwöhnungsbehandlungen werden strukturelle Aspekte der Behandlung von Abhängigkeitserkrankungen in Einrichtungen der medizinischen Rehabilitation beschrieben. Die von der Rentenversicherung routinemäßig erhobene Reha-Statistik zeigt, dass die Anzahl der durchgeführten Entwöhnungsbehandlungen in den letzten Jahren kontinuierlich gestiegen ist. Einerseits ist dieser Trend - im Gegensatz zur Entwicklung in anderen Indikationsbereichen auch nach Einführung der Spargesetze im Jahre 1997 ungebrochen. Andererseits ist bei Entwöhnungsbehandlungen seit 1997 eine deutliche Verkürzung der Rehabilitationsdauer zu verzeichnen. Die Darstellung struktureller Aspekte der Entwöhnungsbehandlung basiert auf Ergebnissen des Reha-Qualitätssicherungsprogramms der Rentenversicherung. Untersucht werden strukturelle Unterschiede zwischen Einrichtungen für die Behandlung von Alkohol- und Medikamentenabhängigkeit und Einrichtungen für die Behandlung der Abhängigkeit von illegalen Drogen. Zum anderen erfolgt innerhalb der genannten Gruppen ein Vergleich zwischen Fachabteilungen, die an größere Häuser angegliedert sind, und eigenständig arbeitenden Entwöhnungseinrichtungen. Insbesondere hinsichtlich des Vergleichs von Fachabteilungen und eigenständigen Einrichtungen werden strukturelle Unterschiede sichtbar.

Schlüsselwörter: Medizinische Rehabilitation - Abhängigkeitserkrankungen - Strukturqualität - Personalstruktur Therapeutische Leistungen

In-Patient Medical Rehabilitation for Patients with Alcohol and Drug Dependence - Structural Aspects: Based on an overview on rehabilitation statistics for the therapy of drug and alcohol dependence recorded by the German pension insurance, this article illustrates structural aspects of the dependency treatment in medical rehabilitation centers. The routine statistics of the German pension insurance indicates that the number of dependency treatments has been continuously increasing within the last years. On one hand, unlike for other indications, this trend is still ongoing - despite the political economy measures of 1997 . On the other hand, duration of treatment has significantly decreased since 1997.

Suchttherapie 2000; 1: 110-116

(c) Georg Thieme Verlag Stuttgart · New York

ISSN 1439-9903
The description of structural aspects of dependency treatment is based on results from the rehabilitation quality management program of the German pension insurance and focuses on structural differences between centers for the treatment of alcohol and legal drug dependence and centers for illegal drug addicts. Furthermore, in both groups departments affiliated to larger hospitals are compared to autonomous institutions. Particularly between these two types of institutions, structural differences can be found.

Key words: Medical Rehabilitation - Dependency - Structural Quality - Group Therapy - Individual Therapy - Personnel Resources

\section{Umfang der Rehabilitationsleistungen bei Abhängigkeitserkrankungen}

Die nachfolgenden Ausführungen zeigen, dass die Rehabilitation von Abhängigkeitserkrankungen im Bereich der gesetzlichen Rentenversicherung (GRV) bezogen auf die Anzahl der durchgeführten Maßnahmen und insbesondere bezogen auf die entsprechenden Ausgaben einen relativ hohen Stellenwert einnimmt. Dieser Stellenwert hat nach In-Kraft-Treten der Spargesetze (im Wesentlichen Wachstums-und-Beschäftigungs-Förderungsgesetz, WFG) zum 1. Januar 1997 noch zugenommen.

Tab. 1 Entwöhnungsbehandlungen der GRV. Zeitreihe von 1994 bis 1999. Quelle: VDR-Statistik Rehabilitation, Jahrgänge 1994-1999, Einlegeblätter u. Tab. 3A

\begin{tabular}{lllll}
\hline & $\begin{array}{l}\text { Reha- } \\
\text { Leistungen } \\
\text { insgesamt }\end{array}$ & $\begin{array}{l}\text { Entwöh- } \\
\text { nungsbe- } \\
\text { handlungen* }\end{array}$ & $\begin{array}{l}\text { Entwöh- } \\
\text { nungs- } \\
\text { behand- } \\
\text { lungen in \% }\end{array}$ & $\begin{array}{l}\text { Steigerung } \\
\text { zum Vorjahr } \\
\text { in \% }\end{array}$ \\
\hline 1994 & 913724 & 32612 & 3,7 & 6,9 \\
1995 & 928295 & 34029 & 3,8 & 4,3 \\
1996 & 960622 & 35090 & 3,7 & 3,1 \\
1997 & 629752 & 41468 & 6,6 & 18,2 \\
1998 & 642436 & 42680 & 6,6 & 2,9 \\
1999 & 717388 & 43793 & 6,1 & 2,6 \\
\hline
\end{tabular}

* ab 1997: ambulante u. stationäre Entwöhnungsbehandlungen 
Tab.2 Mittlere Behandlungstage bei stationären medizinischen und sonstigen Leistungen zur Rehabilitation der Rentenversicherung 1994-1999. Quelle: VDR-Statistik Rehabilitation, Jahrgänge 1994-1999, Tab. 7.00M, eigene Berechnungen

\begin{tabular}{|c|c|c|c|c|c|c|c|}
\hline Diagnosegrundgruppen & 1994 & 1995 & 1996 & 1997 & 1998 & 1999 & $\begin{array}{l}\text { Verände- } \\
\text { rung } 1996 \\
\text { zu } 1999\end{array}$ \\
\hline insgesamt & 35 & 34 & 33 & 33 & 32 & 31 & $6,1 \%$ \\
\hline insgesamt (ohne Sucht) & 32 & 31 & 30 & 29 & 28 & 27 & $10,0 \%$ \\
\hline Krankheiten der Nerven- und Sinnesorgane & 37 & 36 & 35 & 33 & 33 & 32 & $8,6 \%$ \\
\hline Krankheiten von Skelett, Muskeln, Bindegewebe & 30 & 30 & 28 & 25 & 25 & 25 & $-10,7 \%$ \\
\hline Stoffwechsel, Verdauungsorgane & 31 & 30 & 30 & 28 & 25 & 25 & $-16,7 \%$ \\
\hline Krankheiten des Kreislaufsystems & 31 & 30 & 30 & 28 & 26 & 26 & $-13,3 \%$ \\
\hline Krankheiten der Haut & 32 & 32 & 30 & 27 & 27 & 24 & $-20,0 \%$ \\
\hline Krankheiten der Atmungsorgane & 30 & 29 & 28 & 25 & 24 & 24 & $-14,3 \%$ \\
\hline psychische Erkrankungen (ohne Sucht) & 41 & 42 & 43 & 43 & 41 & 41 & $4,7 \%$ \\
\hline Neubildungen & 30 & 30 & 29 & 26 & 25 & 25 & $-13,8 \%$ \\
\hline \multicolumn{8}{|l|}{ Sucht: } \\
\hline Alkoholabhängigkeit & 110 & 110 & 108 & 96 & 88 & 87 & $-19,4 \%$ \\
\hline Medikamenten-/Drogenabhängigkeit & 140 & 146 & 149 & 135 & 119 & 119 & $-20,1 \%$ \\
\hline
\end{tabular}

Die Entwicklung der durchgeführten medizinischen Rehabilitationsleistungen insgesamt zeigt seit 1994 einen unsteten Verlauf: Von 1994 bis 1996 ist eine kontinuierliche Zunahme auf 960622 Maßnahmen zu verzeichnen (vgl. Tab. 1). Im Jahr des In-Kraft-Tretens der Spargesetzgebung, 1997, erfolgt ein drastischer Rückgang um rund 34\% auf einen Tiefpunkt von 629752 Maßnahmen. In den Jahren 1998 und 1999 kann wieder ein Anstieg festgestellt werden, wobei die Anzahl der Rehabilitationsleistungen 1999 mit 717388 Maßnahmen immer noch deutlich unter dem Niveau vor dem In-Kraft-Treten der Spargesetze liegt. Bei der Entwicklung der Anzahl der Entwöhnungsbehandlungen zeigt sich ein anderes Bild: Hier ist im Zeitraum von 1994 bis 1999 eine kontinuierliche Zunahme der Reha-Leistungen von 32612 auf 43793 RehaLeistungen festzustellen. Dabei ist der starke Anstieg von 1996 auf 1997 zum Teil dadurch zu erklären, dass die ambulanten Entwöhnungsbehandlungen erst seit 1997 systematisch erfasst wurden. Der Anteil der ambulant durchgeführten Entwöhnungsbehandlungen ist in den letzten Jahren auf rund 15\% im Jahr 1999 gestiegen. Als Folge der unterschiedlichen Entwicklungsverläufe bei den gesamten Reha-Leistungen und den Entwöhnungsbehandlungen ist der Anteil der Entwöhnungsbehandlungen an den Reha-Leistungen insgesamt von 3,7\% im Jahr 1996 auf über 6\% in den Folgejahren gestiegen. Dabei liegt dieser Anteil in der Arbeiterrentenversicherung traditionell höher als in der Angestelltenversicherung (1999: $\mathrm{ArV}=8,3 \%, \mathrm{AnV}=3,4 \%$ ). Weiterhin zeigt sich ein geschlechtsspezifischer Unterschied, da der Anteil der Männer bei den Entwöhnungsbehandlungen mit $81 \%$ (1999) deutlich höher ausfällt als bei den gesamten Reha-Leistungen mit 55\% (1999).

Die durchschnittliche Dauer der Rehabilitationsmaßnahmen zeigt, bezogen auf alle (stationären) Rehabilitationsmaßnahmen ohne Suchterkrankungen von 1994 (32 Behandlungstage) bis 1999 (27 Behandlungstage), eine kontinuierliche jährliche Abnahme um jeweils einen Behandlungstag (vgl. Tab. 2). Bei den Rehabilitationsleistungen wegen Alkoholabhängigkeit kann nach einer Reha-Dauer von jeweils 110 Behandlungstagen in den Jahren 1994 und 1995 eine Verkürzung der Reha-Dauer festgestellt werden, wobei diese von
1996 auf 1997 mit einem Rückgang um 12 Behandlungstage besonders stark ausfällt. 1999 liegt die durchschnittliche Reha-Dauer hier bei 87 Tagen. Die durchschnittliche Dauer bei Maßnahmen wegen Medikamenten-/Drogenabhängigkeit steigt von 1994 bis 1996 auf 149 Tage an, um danach in großen Sprüngen auf 135 Tage (1997) und 119 Tage (1998 und 1999) abzunehmen. Ein Vergleich der durchschnittlichen Reha-Dauer bei den verschiedenen Indikationsgruppen für die Jahre 1996 und 1999 ergibt, dass der relative Rückgang (Differenz zwischen 1999 und 1996 bezogen auf die Dauer 1996) bei den Indikationen „Medikamenten-/Drogenabhängigkeit“ (20,1\%), „Krankheiten der Haut“ (20,0\%) und „Alkoholabhängigkeit“ (19,4\%) am stärksten ausgeprägt ist.

Die finanziellen Aufwendungen für medizinische und ergänzende $^{1}$ Reha-Leistungen bei Suchterkrankungen werden wesentlich beeinflusst durch die Anzahl der durchgeführten Reha-Maßnahmen sowie die Rehabilitationsdauer. Bei den entsprechenden Aufwendungen für Suchterkrankungen ist von 1994 bis 1996 ein Anstieg auf rund 940 Millionen DM zu verzeichnen (vgl. Tab.3). Der Rückgang der Ausgaben auf rund 830 Millionen im Jahr 1997 kann im Wesentlichen durch die relativ starke Reduzierung der Rehabilitationsdauer der Entwöhnungsbehandlungen von 1996 auf 1997 erklärt werden (s.o.). Nach 1997 zeigt sich eine Zunahme der Ausgaben auf rund 873 Millionen DM im Jahr 1999. Die Entwöhnungsbehandlungen nehmen bei den Aufwendungen traditionell einen deutlich höheren Stellenwert ein, als dies ihrem mengenmäßigen Anteil entsprechen würde. Dies kann im Wesentlichen auf die vergleichsweise lange Rehabilitationsdauer bei Entwöhnungsbehandlungen zurückgeführt werden. Dabei liegt der Anteil der Aufwendungen für Suchterkrankungen an den Gesamtaufwendungen nach In-KraftTreten der Spargesetze mit über 16\% deutlich höher als vorher (1996: 12,5\%).

\footnotetext{
${ }^{1} \mathrm{Zu}$ den ergänzenden Leistungen zählen z.B. Übergangsgeld, Haushaltshilfe und Reisekosten. In der Statistik der finanziellen Aufwendungen wurden die Aufwendungen für ambulante Leistungen schon vor 1997 einbezogen.
} 
Tab. 3 Bruttoaufwendungen in Mio. DM für medizinische und ergänzende Reha-Leistungen wegen Abhängigkeitserkrankungen $1994-1999$. Quelle: VDR-Statistik Rehabilitation, Jahrgänge 1994 - 1999, Einlegeblätter

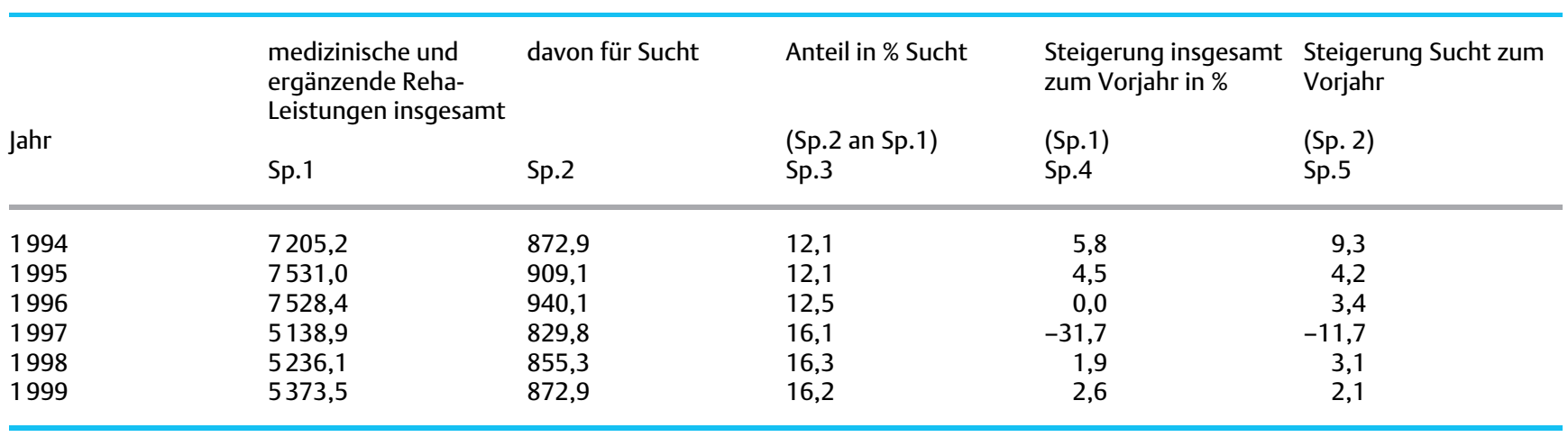
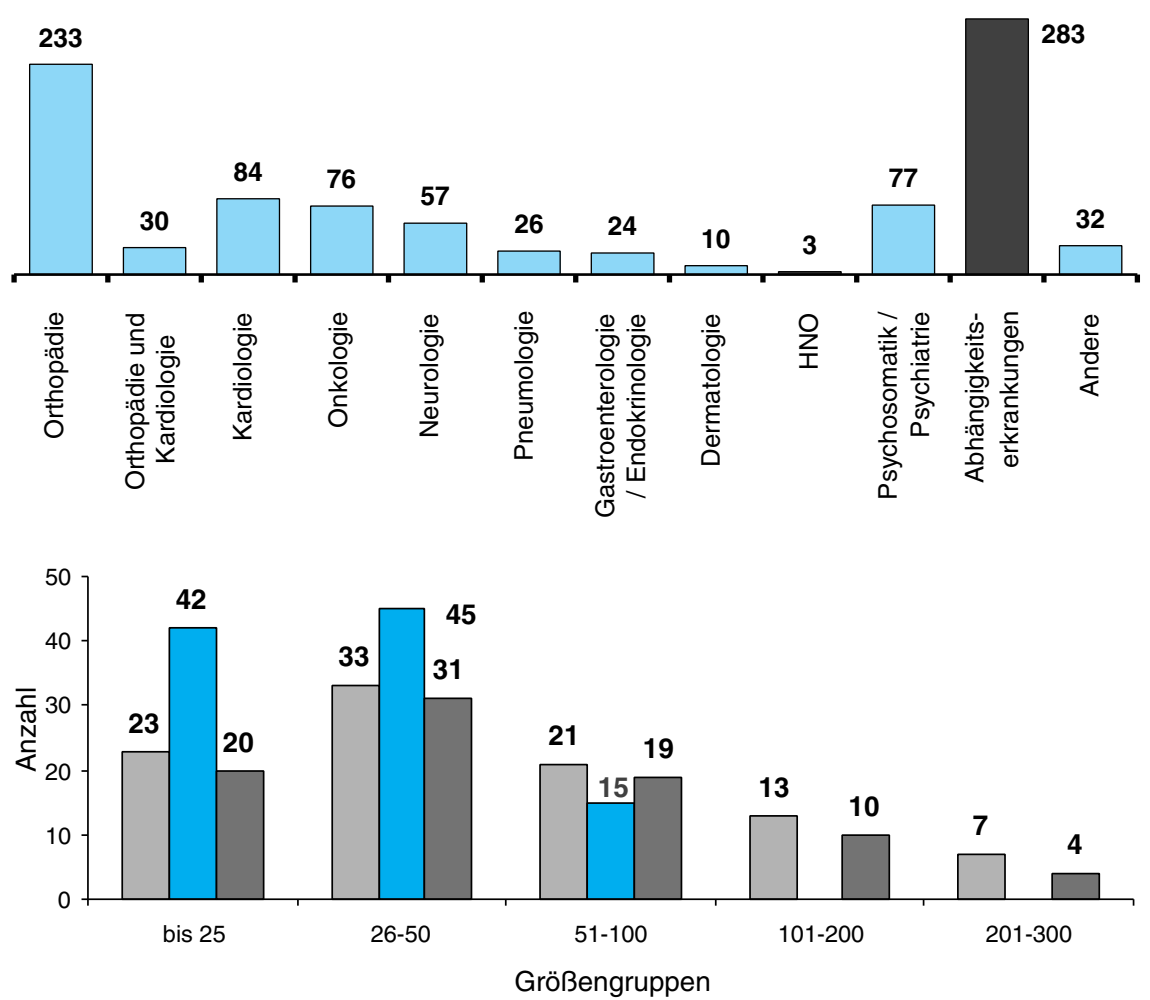

Abb. 1 Rehabilitationseinrichtungen im Qualitätssicherungsprogramm der Rentenversicherung $(n=935)$.

Abb. 2 Einrichtungsgröße $(n=283)-$ Einrichtungen für Abhängigkeitserkrankungen.

$\square$ Alkohol / Medikamente $\square$ Illegale Drogen $\square$ Beides

\section{Struktur der Rehabilitationseinrichtungen}

Am Qualitätssicherungsprogramm der Rentenversicherung2 nehmen 935 Rehabilitationseinrichtungen teil. Dazu gehören 283 Einrichtungen für Abhängigkeitserkrankungen (vgl. Abb. 1). Diese verfügen insgesamt über 13.987 Behandlungsplätze. Der größere Teil der Einrichtungen $(n=231)$, der $68 \%$ der Behandlungsplätze $(n=9545)$ einschließt, wird federführend von der Arbeiterrentenversicherung belegt. Bei 52 Einrichtungen resp. $32 \%$ der Behandlungsplätze $(n=4442)$ ist die Angestelltenversicherung federführend. 78 der 283 Einrichtungen für Abhängigkeitserkrankungen bieten neben der stationären Behandlung auch ambulante/teilstationäre Leis-

\footnotetext{
${ }^{2}$ Vgl. Egner et al. 1998 einschl. der dort angegebenen Literatur
}

tungen an. Hierfür sind zusätzlich 1257 Behandlungsplätze vorgesehen.

Während die durchschnittliche Größe der somatischen und psychosomatischen Einrichtungen bei 154 Betten bzw. Behandlungsplätzen liegt, beträgt die durchschnittliche Größe der Einrichtungen für Abhängigkeitserkrankungen 48. Die Einrichtungen dieses Indikationsbereichs sind im Durchschnitt erheblich kleiner als diejenigen der anderen Indikationsbereiche.

Die 283 Einrichtungen für Abhängigkeitserkrankungen im Qualitätssicherungsprogramm können in drei Gruppen unterteilt werden. Dabei handelt es sich um Einrichtungen für Alkohol- und Medikamentenabhängigkeit $(n=97)$, Einrichtungen für die Behandlung der Abhängigkeit von illegalen Drogen $(n=102)$ und Einrichtungen, die Rehabilitationsleis- 
tungen sowohl bei Alkohol- und Medikamentenabhängigkeit als auch bei Abhängigkeit von illegalen Drogen durchführen $(\mathrm{n}=84)$.

$22 \%$ ( $n=21)$ der insgesamt 97 Einrichtungen für Alkoholund Medikamentenabhängige sind Fachabteilungen größerer Einrichtungen. Bei den Einrichtungen für Drogenabhängige beträgt dieser Anteil lediglich 6\% $(n=6)$. In den Einrichtungen, die Rehabilitationsleistungen sowohl bei Alkohol- und Medikamentenabhängigkeit als auch bei Abhängigkeit von illegalen Drogen durchführen, liegt er bei $8 \%(n=7)$. In aller Regel handelt es sich um Fachabteilungen in psychatrischen Landeskliniken mit den entsprechenden Spezifika dieser Einrichtungen.

Es zeigt sich, dass insbesondere die Behandlung der Abhängigkeit von illegalen Drogen in kleinen Einrichtungen durchgeführt wird. Über $85 \%(n=87)$ dieser Einrichtungen halten maximal 50 Behandlungsplätze vor (vgl. Abb. 2). Bei Einrichtungen für die Behandlung von Alkohol- und Medikamentenabhängigkeit beträgt dieser Anteil nur knapp 58\% $(n=56)$. In der dritten Gruppe, den Einrichtungen, die Entwöhnungsbehandlungen sowohl bei Alkohol- und Medikamentenabhängigkeit als auch bei Abhängigkeit von illegalen Drogen durchführen, liegt der Anteil der Einrichtungen mit maximal 50 Behandlungsplätzen bei $61 \%(n=51)$.

Wie im Folgenden noch gezeigt wird, weisen die beiden erstgenannten Gruppen teilweise deutliche strukturelle Unterschiede auf. Die strukturellen Ausprägungen der dritten Gruppe (Behandlung der Alkohol- und Medikamentenabhängigkeit und der Abhängigkeit von illegalen Drogen) liegen hingegen regelmäßig innerhalb des Wertebereichs der anderen beiden. Es ist davon auszugehen, dass in dieser Gruppe eine auf die Art der Abhängigkeitserkrankung bezogene Binnenstrukturierung vorliegt (z.B. bezogen auf die Teamorganisation und auf die Personalstrukturen). Aus diesem Grunde wird im Weiteren auf eine Untersuchung dieser Gruppe verzichtet.

Bei einem Vergleich der Einrichtungen für Alkohol- und Medikamentenabhängigkeit und der Einrichtungen für Drogenabhängigkeit zeigen sich hinsichtlich der Personalstrukturen teilweise erhebliche Unterschiede. Entsprechende Unterschiede ergeben sich auch, wenn innerhalb der beiden Gruppen Fachabteilungen und eigenständige Häuser gegenübergestellt werden (vgl. Abb. 3 u. 4). Bei der Interpretation ist zu berücksichtigen, dass der Anteil der Fachabteilungen bei den Einrichtungen für die Behandlung der Abhängigkeit von illegalen Drogen mit $\mathrm{n}=6$ relativ klein ist.

Ärzte, Pflegekräfte und physiotherapeutische Dienste haben im Rehabilitationsteam der Einrichtungen für Alkohol- und Medikamentenabhängige ein höheres Gewicht als in den Einrichtungen für Drogenabhängige. Auffällig ist der hohe Anteil an Pflegekräften (15,6 Pflegekräfte je 100 Betten) in den Fachabteilungen für Alkohol- und Medikamentenabhängige (vgl. Abb.3). Traditionell wird in Landespsychiatrien das Pflegepersonal mit entsprechender Zusatzqualifikation als therapeutisches Personal eingesetzt. Hiermit lässt sich im Wesentlichen der hohe Anteil an Pflegekräften in den Fachabteilungen erklären.

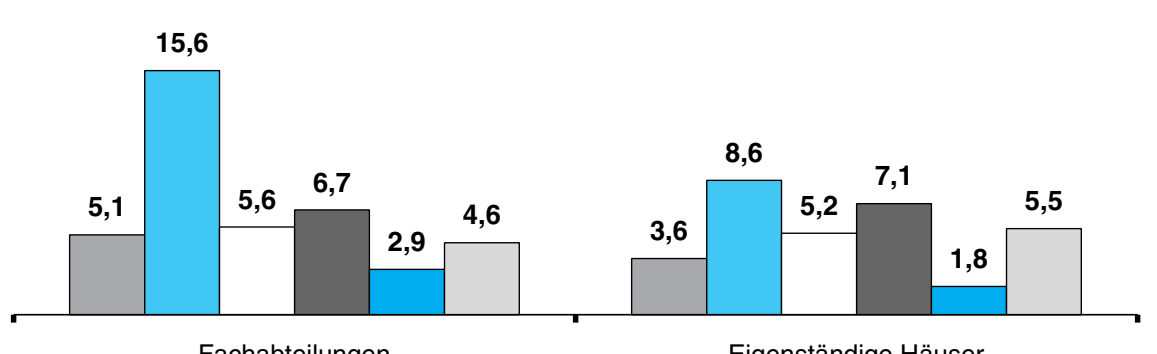

Fachabteilungen

Eigenständige Häuser

\begin{tabular}{|ll|}
\hline Ärztlicher Dienst & $\square$ Pflegedienst \\
$\square$ Psychologischer Dienst & $\square$ Sozialer und pädagogischer Dienst \\
$\square$ Physioth. Dienste & $\square$ Weitere therap. Dienste \\
\hline
\end{tabular}

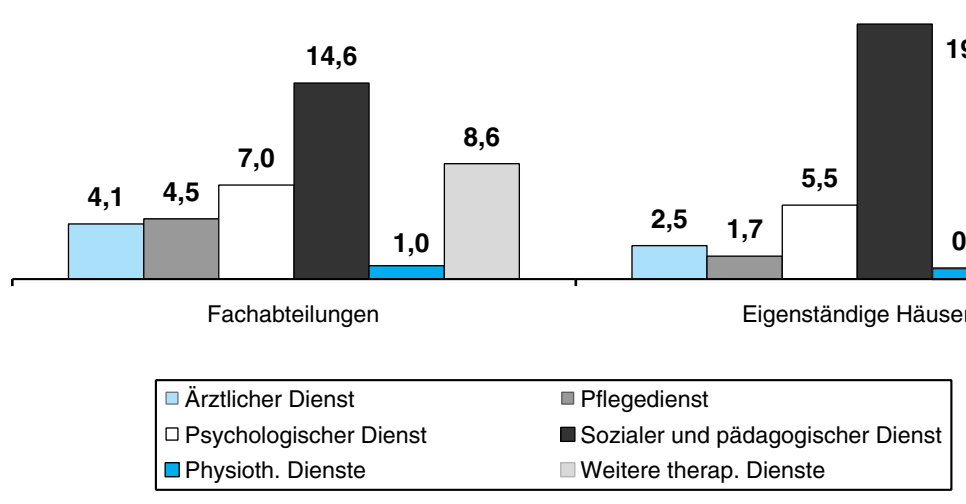

Abb. 3 Einrichtungen für Alkohol- und Medikamentenabhängigkeit - Personaldichte in Fachabteilungen $(n=21)$ und eigenständigen Einrichtungen $(n=76)$ - Personal je 100 Betten, um den Einfluss von Extremwerten zu minimieren, wurde als Maß der Medien gewählt.
6,9 


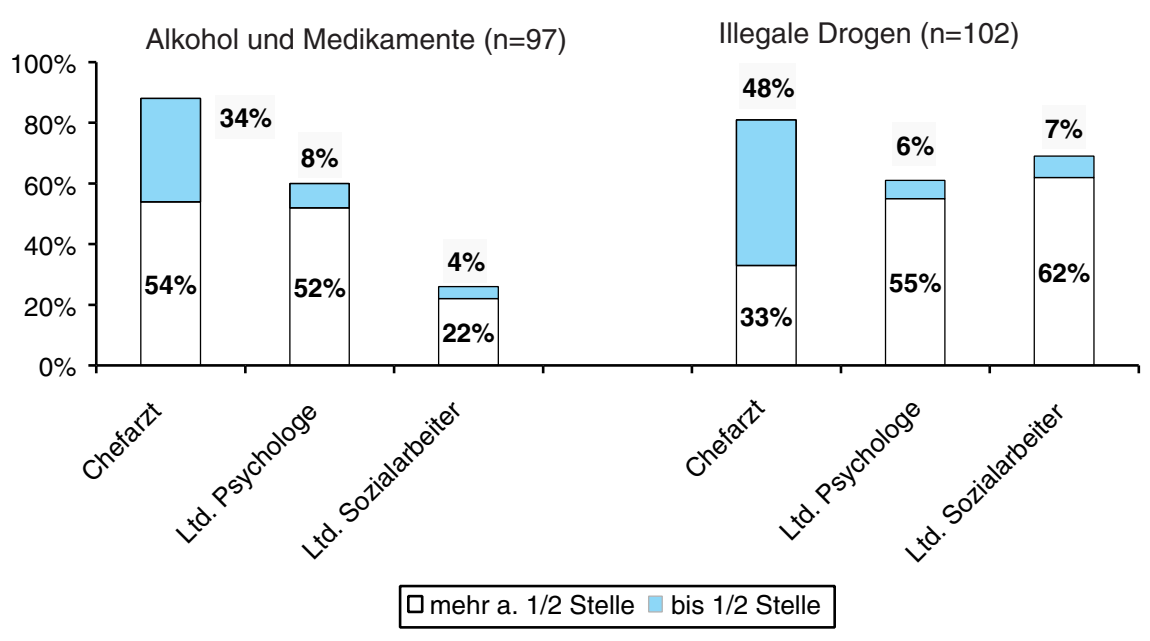

Abb. 5 Leitende Berufsgruppen in Einrichtungen für Abhängigkeitserkrankungen (Alkohol/Medikamente vs. illegale Drogen).

Hervorzuheben ist auch die Bedeutung der Sozialarbeiter im Rehabilitationsteam der Einrichtungen für Drogenabhängige (vgl. Abb. 4). Die Personaldichte dieser Berufsgruppe beträgt in Fachabteilungen 14,6 und in eigenständigen Häusern sogar 19 Sozialarbeiter auf 100 Behandlungsplätze. Weitestgehend sind dies Mitarbeiter, die nach der Empfehlungsvereinbarung auf der Grundlage entsprechender Zusatzqualifikationen als Therapeuten eingesetzt werden. Eine den Einrichtungen für Alkohol- und Medikamentenabhängigkeit gegenüber vergleichsweise hohe Bedeutung haben in den Einrichtungen für Drogenabhängige auch ergo- und kreativtherapeutische Dienste, die hier mit 8,6 bzw. 6,9 Stellen auf 100 Behandlungsplätze als weitere therapeutische Dienste ausgewiesen werden.

Die berufsgruppenbezogenen Unterschiede zwischen den Einrichtungen für Alkohol- und Medikamentenabhängigkeit und den Einrichtungen für die Behandlung der Abhängigkeit von illegalen Drogen spiegelt sich auch in der Besetzung der leitenden Positionen wider (vgl. Abb. 5). Die hier untersuchten Drogeneinrichtungen verfügen $\mathrm{zu} 62 \%$ über leitende Sozialarbeiter, die mehr als halbschichtig in den Einrichtungen tätig sind. Demgegenüber beschäftigen nur 26\% der Einrichtungen für Alkohol- und Medikamentenabhängige überhaupt leitende Sozialarbeiter. Der Anteil mehr als halbschichtig tätiger Chefärzte in Drogeneinrichtungen ist mit $33 \%$ niedriger als in Einrichtungen für Alkohol- und Medikamentenabhängige (54\%). Bei der Bewertung ist zu berücksichtigen, dass in den Einrichtungen durchaus auch parallel Chefarzt, leitende Psychologen und leitende Sozialarbeiter beschäftigt sein können. Wie aus Abb. 5 ersichtlich, verfügen $88 \%$ der Einrichtungen für Alkohol- und Medikamentenabhängigkeit über einen Chefarzt. In den übrigen $12 \%$ dieser Einrichtungen wird die Leitung bei einem Psychologen oder einem Sozialarbeiter liegen.

Bei der Untersuchung der therapeutischen Angebote konnten weder hinsichtlich der Unterscheidung der Einrichtungen für Alkohol- und Medikamentenabhängigkeit und der Einrichtungen für Drogenabhängigkeit noch hinsichtlich der Unter-

\footnotetext{
3 Vgl. VDR 2000
}

scheidung zwischen den Fachabteilungen und den eigenständigen Einrichtungen klar differenzierende Sets gefunden werden. Dies wird hier am Beispiel der Einrichtungen für Alkohol- und Medikamentenabhängigkeit gezeigt (vgl. Abb.6). Sowohl in den Fachabteilungen als auch in den eigenständigen Häusern werden alle im Strukturbogen ${ }^{3}$ erhobenen einzeltherapeutischen Verfahren durchgeführt, wenn auch mit leicht unterschiedlicher Gewichtung. Die Gewichte liegen dabei auf den verhaltenstherapeutischen, gesprächspsychotherapeutischen und tiefenpsychologischen Ansätzen gleichermaßen. In den Fachabteilungen scheinen psychoanalytisch orientierte Therapieformen (z.B. tiefenpsychologisch orientierte Einzeltherapie, psychoanalytische Einzeltherapie, ich-strukturelle Einzeltherapie) leicht zu überwiegen. Andererseits finden Organisation und Monitoring der internen und externen Belastungserprobung in den eigenständigen Häusern eine größere Verbreitung.

Die Daten wurden auch auf Muster untersucht, die Hinweise darauf zulassen könnten, dass bezogen auf den Einrichtungstyp (Fachabteilung vs. eigenständige Häuser) oder bezogen auf die Substanz (Alkohol/Medikamente vs. illegale Drogen) Unterschiede hinsichtlich der Therapieform bzw. der therapeutischen Schule bestehen. Betrachtet man beispielhaft die Einrichtungen für Alkohol- und Medikamentenabhängigkeit, zeigt sich, dass im Rahmen einzeltherapeutischer Leistungen in $38 \%(n=37)$ der Einrichtungen und Fachabteilungen nebeneinander tiefenpsychologische, verhaltenstherapeutische und gesprächspsychotherapeutische Verfahren und in $33 \%(n=32)$ der Einrichtungen zwei der genannten Therapieformen zum Einsatz kommen (vgl. Abb. 7). In 27\% ( $n=26)$ der Einrichtungen wird lediglich eine der genannten Therapieformen angewandt. In 19 der 26 Einrichtungen ist dies die tiefenpsychologisch fundierte Einzeltherapie.

Wie bei den einzeltherapeutischen Angeboten ist auch das Spektrum der gruppentherapeutischen Leistungen vielfältig. Exemplarisch bildet Abb. 8 die Leistungen ab, die in über $50 \%$ der Einrichtungen für Alkohol- und Medikamentenabhängigkeit (eigenständige Einrichtungen und Fachabteilungen zusammengenommen) angeboten werden. Daneben haben ergo- und arbeitstherapeutische Leistungen eine herausragende Bedeutung in der Rehabilitation bei Alkohol- und Medika- 
mentenabhängigkeit (z.B. Hauswirtschaft 79\%, Gärtnerei 78\%, Küchendienste 77\%, Schreinerei 76\%, Schlosserei 70\%).

Innerhalb der Gruppe der hier untersuchten Einrichtungen für Alkohol- und Medikamentenabhängigkeit bieten die Fachabteilungen stärker als die eigenständigen Einrichtungen neben den einzel- und gruppentherapeutischen Verfahren auch Leistungen wie Krankengymnastik sowie Sport- und Bewegungstherapie an (z.B. Lauf- und Gehtraining: Fachabteilung 95\%, eigenständige Einrichtungen 84\%; Muskelaufbautraining: 90\%, 75\%; Schwimmen: 90\%, 75\%; Koordinations- und Gleichgewichtstraining: 90\%, 67\%; Sportthe- rapie: 71\%, 61\%; Atemgymnastik: 71\%, 58\%). Dies korrespondiert mit den in Abb. 3 vorgestellten Personalzahlen.

Von herausragender Bedeutung in den Einrichtungen für Abhängigkeitserkrankungen insgesamt sind außerdem die Beratungsleistungen. Die wichtigsten Beratungsleistungen stellen das Bewerbungstraining, Beratungsangebote $\mathrm{zu}$ speziellen Rehabilitationsleistungen, zur finanziellen Sicherstellung und zur Nachsorge dar, die jeweils in über $80 \%$ der Einrichtungen angeboten werden (vgl. Abb.9). Drogeneinrichtungen bieten zusätzlich stärker als Einrichtungen für die

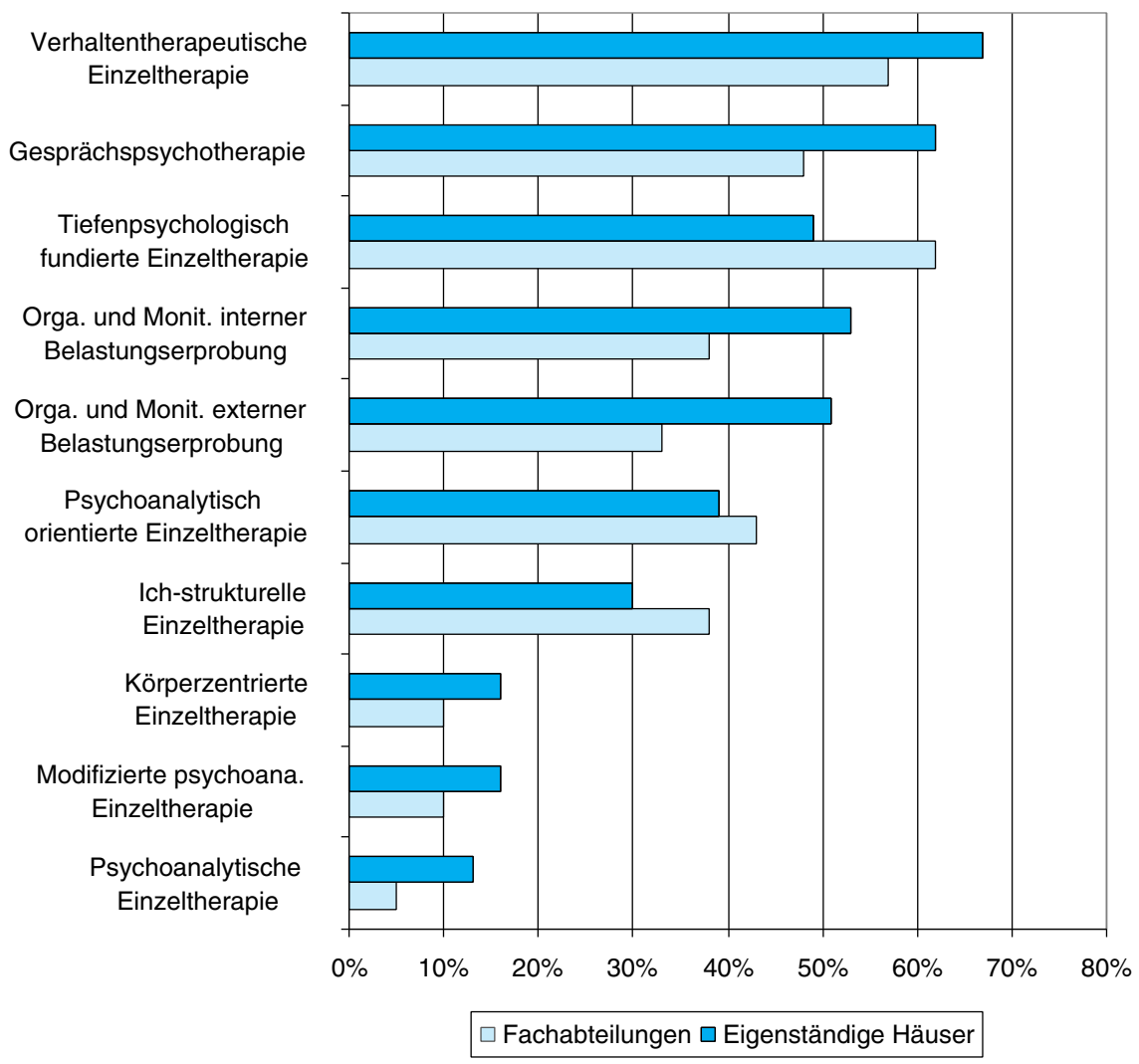

Abb. 6 Einzeltherapeutische Leistungen in Fachabteilungen $(n=21)$ und eigenständigen Einrichtungen ( $n=76$ ) für Alkohol- und Medikamentenabhängigkeit.

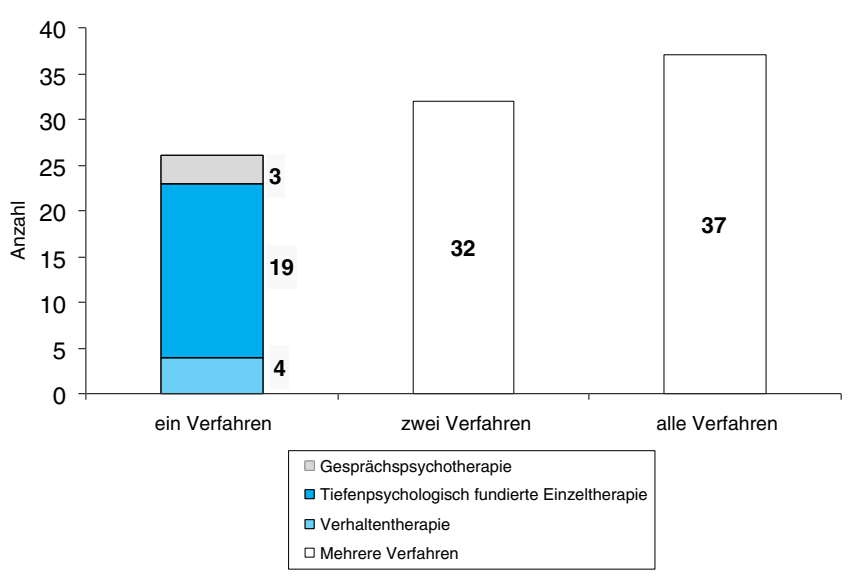

Abb. 7 Einzeltherapieformen in Einrichtungen für Alkohol- und Medikamentenabhängigkeit ( $n=97,2$ missings).
Behandlung von Alkohol- und Medikamentenabhängigkeit auf Beruf und Erwerbstätigkeit bezogene Beratungsleistungen an (z.B. Berufsfindung, Praktikumsvermittlung).

Die Angaben der am Qualitätssicherungsprogramm beteiligten Rehabilitationseinrichtungen für Abhängigkeitserkrankungen korrespondieren mit den Angaben der Patienten in der regelmäßig durchgeführten Patientenbefragung. Hierzu wurden die Angaben von Versicherten der Arbeiterrentenversicherung mit entsprechender Hauptdiagnose herangezogen, die zwischen Oktober 1999 und März 2000 im Rahmen der Patientenbefragung im Qualitätssicherungsprogramm befragt wurden $(n=1738)$. Danach nahmen alle Patienten an gruppentherapeutischen Verfahren teil. 94\% erhielten einzeltherapeutische Leistungen. Ein ebenso großer Anteil der Patienten nahm an sport- und bewegungstherapeutischen Angeboten teil. Von herausragender Bedeutung sind laut Patientenangaben auch die Ergo-, Arbeits- und Beschäftigungstherapie. Demgegenüber wird das breite Angebot an 

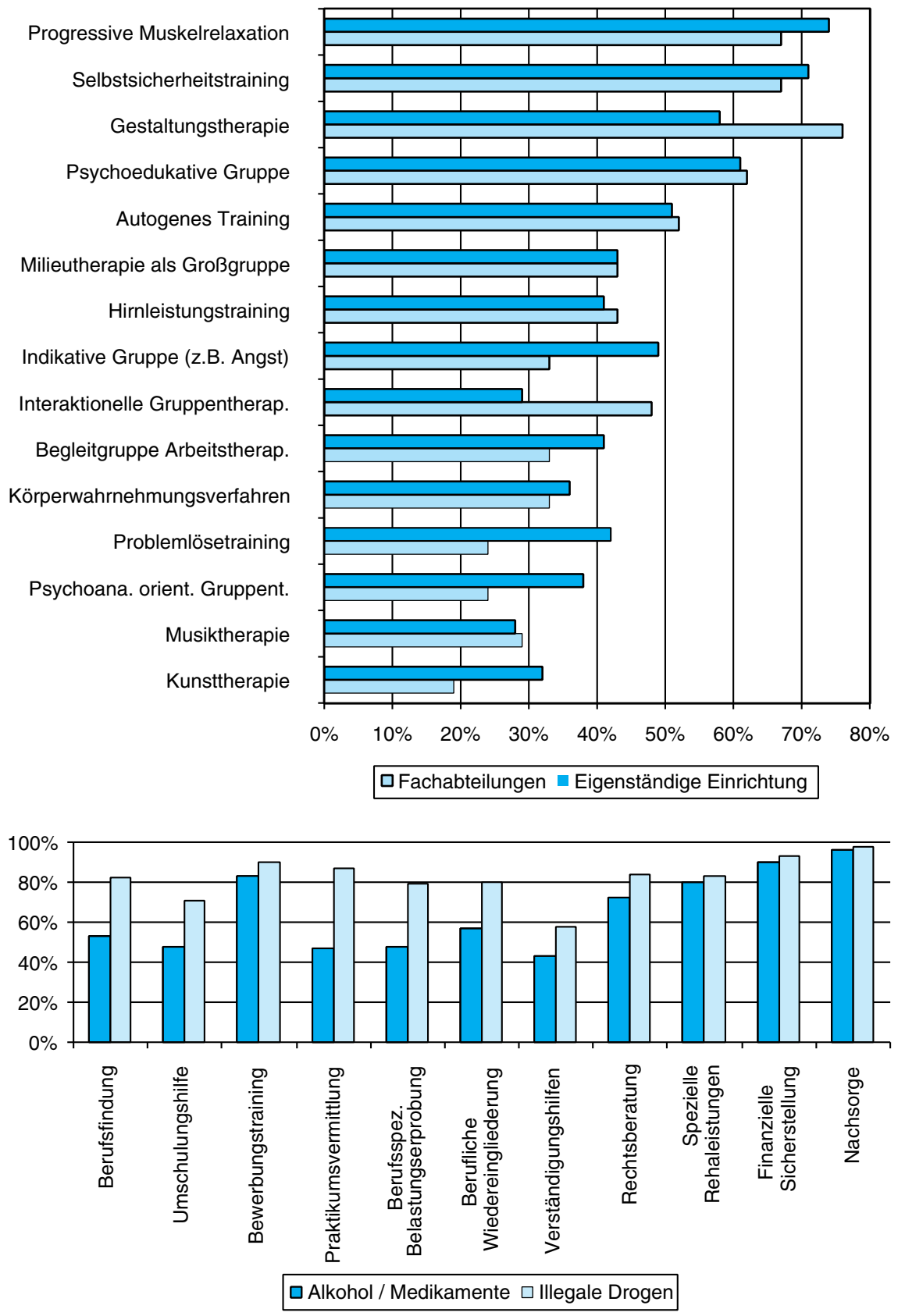

Abb. 8 Gruppentherapeutische Leistungen in Fachabteilungen $(n=21)$ und eigenständigen Einrichtungen $(n=76)$ für Alkohol- und Medikamentenabhängigkeit.

Abb. 9 Beratungsleistungen in Einrichtungen für Abhängigkeitserkrankungen $(n=199)$
Beratungsleistungen vergleichsweise wenig genutzt. Nur $28 \%$ der Alkohol- oder Medikamentenabhängigen und 48\% der Abhängigen von illegalen Drogen gaben an, spezielle Beratungsleistungen in Anspruch genommen zu haben. Neben den Angaben zu den therapeutischen Leistungen haben die befragten Patienten Gelegenheit, sich über ihre Zufriedenheit mit der Rehabilitationsleistung insgesamt zu äußern. 75\% der Patienten gaben an, mit der Rehabilitationsleistung insgesamt zufrieden oder sehr zufrieden zu sein.

\section{Literatur}

${ }^{1}$ Egner U, Gerwinn H, Müller-Fahrnow W, Schliehe F. Das Qualitätssicherungsprogramm der gesetzlichen Rentenversicherung für den Bereich der medizinischen Rehabilitation. Die Rehabilitation 1998; 37, Supplement 1: S2-S7
${ }^{2}$ Verband Deutscher Rentenversicherungsträger. Das Qualitätssicherungsprogramm der gesetzlichen Rentenversicherung in der medizinischen Rehabilitation - Instrumente und Verfahren. Selbstverlag, 2000

Klaus Huck-Langer

Verband Deutscher Rentenversicherungsträger Eysseneckstraße 55

60322 Frankfurt 